\title{
TECHNOLOGY
}

\section{Let there be light-new implantable hydrogels for in vivo optical sensing and therapy}

Researchers from the USA and South Korea have developed a medical implant, a so-called light-guiding hydrogel, that can function as a cellbased sensor (for example, of toxins) or therapeutic device. Hydrogel is a wellknown biomaterial and has previously been used for drug delivery; however, its use as an optical material to enable the delivery of light to cells is unprecedented.

The implant comprises a polymerbased, light-guiding scaffold and cells genetically engineered to either emit light in response to a particular metabolic signal (for example, cytotoxic stress) or perform a specific activity (for example, drug delivery) in response to photostimulation. "We made transparent hydrogel into a form of optical waveguide with a fibre-optic pigtail," explains senior investigator Seok-Hyun (Andy) Yun of Harvard Medical School. The optical fibre connects the hydrogel patch to an external light source or detector depending on the function of the implant.
Cell-containing hydrogel patches were implanted into freely moving mice for up to 8 days and shown to offer consistent transparency, optical transmittance, biocompatibility and cell survival. By inserting optogenetic cells into the hydrogel that produce and secrete glucagon-like peptide 1 (GLP-1) in response to photostimulation, the researchers were able to improve glucose homeostasis in streptozotocin-induced diabetic mice in a light-controlled manner.

"This device allows us to send light to the cells in the hydrogel for optogenetic control of protein synthesis and to collect luminescence (fluorescence) from the hydrogel," says Yun, enabling the researchers to establish bidirectional optical communication with cells in vivo.

Yun and colleagues are currently working on different types of hydrogel and cells to improve the production of GLP-1. Furthermore, the researchers are interested in applying light-guiding hydrogel to other diseases, such as cancer. Optical In the future, the hydrogel co-workers could help elucidate complex developmental processes and in vivo disease progression. Hopefully, this technology will also improve drug delivery. "This implant system can potentially replace the inconvenient needle-based injection of protein-based drugs," concludes Yun.

\section{Linda Koch}

Original article Choi, M. et al. Light-guiding hydrogels for cell-based sensing and optogenetic synthesis in vivo. Nat. Photonics doi:10.1038/nphoton.2013.27 